\title{
A Novel Handover Decision Policy for Reducing Power Transmissions in the two-tier LTE network
}

\author{
Dionysis Xenakis, Nikos Passas \\ Department of Informatics and Telecommunications \\ University of Athens, Greece \\ \{nio|passas\}@di.uoa.gr
}

\begin{abstract}
Femtocells are attracting a fast increasing interest nowadays, as a promising solution to improve indoor coverage, enhance system capacity, and lower transmit power. Technical challenges still remain, however, mainly including interference, security and mobility management, intercepting wide deployment and adoption from mobile operators and end users. This paper describes a novel handover decision policy for the two-tier LTE network, towards reducing power transmissions at the mobile terminal side. The proposed policy is LTE backward-compatible, as it can be employed by suitably adapting the handover hysteresis margin with respect to a prescribed SINR target and standard LTE measurements. Simulation results reveal that compared to the widely-adopted strongest cell policy, the proposed policy can greatly reduce the power consumption at the LTE mobile terminals, and lower the interference network-wide.
\end{abstract}

Keywords: LTE; femtocells; handover decision policy; energy efficiency; power consumption; interference management;

\section{INTRODUCTION}

The demand for improved indoor coverage and higher data rates have motivated the deployment of short-range, low-cost, consumer-deployed cellular access points, known as femtocells [1]. Femtocells interconnect standard User Equipment (UE) to the mobile operator's network, via the end user's broadband access backhaul. Even though femtocells can support up to a few users, they embody the functionality of a regular base station which operates within the mobile operator's licensed band. In the Release 10 series of standards for the 3rd Generation Partnership Project (3GPP), the Long Term Evolution (LTE) system provisions the deployment of femtocells [2]. The detailed LTE network architecture, along with the access control and mobility management procedures for closed and hybrid access femtocells can be found in [2].

Femtocell deployment may significantly reduce the mobile operator's energy costs, and greatly lower power transmissions $[3,4]$. The results in [3] indicate that if a femtocell tier is deployed, both the mobile terminals and the cellular stations can reduce their power transmissions by four to eight orders of magnitude. In-band macrocell and femtocell coexistence, however, increases radio-frequency (RF) interference, which in turn degrades the system capacity per-tier and reduces the energy saving potential [4]. Current literature includes various approaches for handling interference in two-tier networks [57], mainly including power control, static or adaptive fractional frequency reuse, spatial techniques, network Multiple Input Multiple Output (MIMO), interference regeneration and cancellation, and intelligent scheduling. Reducing interference

\author{
Christos Verikoukis \\ Telecommunications Technological Centre of Catalonia \\ Barcelona, Spain \\ cveri@cttc.es
}

and power consumption through Mobility Management (MM), however, has not been thoroughly investigated.

MM typically consists of three phases: network discovery, handover (HO) decision, and $\mathrm{HO}$ execution [8]. The $\mathrm{HO}$ decision phase can play a key role in lowering interference and reducing power consumption in the presence of a femtocell tier. Current literature includes various $\mathrm{HO}$ decision algorithms for the two-tier network [9-12], which primarily focus on prioritizing femtocells over macrocells with respect to user mobility criteria. Emphasis is given in reducing the number of the network-wide $\mathrm{HO}$ execution events, owing to the short femtocell radius and the ping-pong effect [8]. Nevertheless, the strongest cell HO decision policy [13] is typically employed for intra-tier HOs, i.e., macrocell to macrocell and femtocell to femtocell HOs. In the context of the two-tier LTE network, however, employing the interference-agnostic strongest cell HO decision policy may result in deteriorated Signal to Interference plus Noise Ratio (SINR), increased network outage probability, and enlarged network signaling owing to false HOs. This negative impact originates from i) the divergent RF interference levels between different cell sites, owing to the random femtocell deployment pattern, and ii) the potentially divergent power transmissions between the Reference Signal (RS) of different femtocells, e.g., when a selfoptimization procedure similar to [14] is employed.

This paper describes a novel HO decision policy for the two-tier LTE network, towards reducing transmit power at the LTE mobile terminals. The employment of the proposed policy is based on adapting the HO Hysteresis Margin (HHM) with respect to a mean SINR target and standard LTE measurements of the candidate cells' status. The incorporation of the SINR target guarantees QoS, while the utilization of standard LTE measurements provides an accurate estimation of the required UE transmit power per candidate cell. The benefit for employing the proposed HO decision policy is three-fold; lower RF interference, reduced power consumption at the LTE UEs, and guaranteed QoS for the ongoing user links. Another important feature of the proposed HO decision policy is that even though it is fundamentally different from the predominant strongest cell HO policy, it is employed in an LTE backwardscompatible manner by suitably adapting the HHM

To the remainder of this paper, Section II includes the adopted system model, and discusses the strongest cell $\mathrm{HO}$ decision policy. Section III describes the proposed HO decision policy, while selected numerical results in Section IV depict the performance of the proposed HO policy, in terms of power 
saving and interference mitigation. Finally, Section V concludes the paper.

\section{SYSTEM MODEL}

\section{A. System description}

A two-tier LTE network is considered, operating within the LTE band set $N:=\{1, \ldots, N\}$. A macrocell station is referred to as evolved Node B (eNB), while a femtocell station as Home eNB (HeNB). An LTE user is member of a Closed Subscriber Group (CSG) if it is permitted to utilize a particular set of closed access femtocells, or if it receives prioritized service from a particular set of hybrid access femtocells [2]. To resourcefully sustain its ongoing services, user $u$ is assumed to have a mean SINR target, denoted by $\bar{\gamma}_{\text {target }}^{u}$.

Let $\boldsymbol{C}_{\boldsymbol{n}}$ denote the set of LTE cells operating in band $n \in \boldsymbol{N}$, including both eNBs and HeNBs, and $\boldsymbol{U}_{\boldsymbol{n}}$ the set of users receiving service from an LTE cell within $\boldsymbol{C}_{\boldsymbol{n}}$. Assuming that user $u \in \boldsymbol{U}_{\boldsymbol{n}}$ is connected to cell $s \in \boldsymbol{C}_{\boldsymbol{n}}$, the respective mean uplink SINR for a tagged time interval $T$ is given as follows:

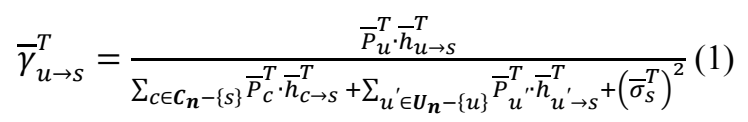

where $\bar{P}_{u}^{T}$ denotes the power transmission of user $u, \bar{h}_{u \rightarrow s}^{T}$ the channel gain from user $u$ to cell $s, \bar{P}_{c}^{T}$ the power transmission of cell $c, \bar{h}_{c \rightarrow s}^{T}$ the channel gain between cells $c$ and $s$, and $\bar{\sigma}_{s}{ }^{2}$ the noise power at cell $s$, all averaged within the time interval $T$. Accordingly, the mean downlink SINR is given as follows:

$$
\bar{\gamma}_{s \rightarrow u}^{T}=\frac{\bar{P}_{s \rightarrow u}^{T} \cdot \bar{h}_{s \rightarrow u}^{T}}{\sum_{c \in C_{n}-\{s\}} \bar{P}_{c}^{T} \cdot \bar{h}_{c \rightarrow u}^{T}+\sum_{u^{\prime} \in \boldsymbol{U}_{\boldsymbol{n}}-\{u\}} \bar{P}_{u}^{T} \cdot \bar{h}_{u^{\prime} \rightarrow u}^{T}+\left(\bar{\sigma}_{u}^{T}\right)^{2}}(2)
$$

where $\bar{P}_{s \rightarrow u}^{T}$ denotes the power transmission of cell $s$ to user $u$, $\bar{h}_{s \rightarrow u}^{T}$ the channel gain from cell $s$ to user $u, \bar{h}_{u^{\prime} \rightarrow u}^{T}$ the channel gain from user $u^{\prime}$ to user $u$, and $\bar{\sigma}_{u}{ }^{2}$ the noise power at user $u$, all averaged within the time interval $T$.

Let us now focus on the expected UE transmit power for maintaining a link between a tagged user $u$ and cell $c$. Let $\boldsymbol{L}_{\boldsymbol{u}} \subseteq \cup_{n \in N} \boldsymbol{C}_{\boldsymbol{n}}$ indicate the candidate cell set for user $u$, which consists of accessible LTE cells and has been identified during the network discovery phase. Using Eq. (1) for the mean SINR target $\bar{\gamma}_{\text {target }}^{u}$, it can be readily shown that the mean UE power transmissions for maintaining a link between user $u$ and cell $c \in \boldsymbol{L}_{\boldsymbol{u}}$ can be estimated as follows:

$$
\bar{P}_{u \rightarrow c}^{T}=\frac{\bar{\gamma}_{\text {target }}^{u} \cdot\left(\sum_{c^{\prime} \in C_{n^{-}\{c\}}} \bar{P}_{c^{T}}^{T} \cdot \bar{h}_{c^{\prime} \rightarrow c}^{T}+\sum_{u^{\prime} \in U_{n^{-}-\{u\}}} \bar{P}_{u^{T}}^{T} \cdot \bar{h}_{u^{\prime} \rightarrow c}^{T}+\left(\bar{\sigma}_{c}^{T}\right)^{2}\right)}{\bar{h}_{u \rightarrow c}^{T}} \text { (3) }
$$

Note that Eq. (3) includes the impact of handing over to cell $c \in \boldsymbol{L}_{\boldsymbol{u}}$, given that the RF interference caused by the ongoing user link, i.e., $\bar{P}_{u}^{T} \cdot \bar{h}_{u \rightarrow s}^{T}$, is not included. Eq. (3) also corresponds to the UE power consumption, owing to transmit power, for maintaining a link between user $u$ and cell $c$. The LTE standard describes a wide set of network and UE link quality measurements [15], which can be utilized to estimate the expected SINR in Eq. (1) and (2), and the average UE power transmission in Eq. (3). Table I summarizes standard

\begin{tabular}{|c|c|c|}
\hline Measurement & Measured by & Notation \\
\hline $\begin{array}{l}\text { Reference Signal Received } \\
\text { Power (RSRP) }\end{array}$ & UE & $R S R P_{c \rightarrow u}^{T}$ \\
\hline $\begin{array}{lcl}\text { E-UTRAN } & \text { Carrier } & \text { Received } \\
\text { Signal } & \text { Strength } & \text { Indicator } \\
\text { (RSSI) } & & \\
\end{array}$ & $\mathrm{UE}$ & $R S S I_{c \rightarrow u}^{T}$ \\
\hline $\begin{array}{l}\text { Reference Signal Received } \\
\text { Quality (RSRQ) }\end{array}$ & $\mathrm{UE}$ & $R S R Q_{c \rightarrow u}^{T}$ \\
\hline $\begin{array}{lll}\text { Downlink } & \text { Reference } & \text { Signal } \\
\text { Transmitted Power (DL RS Tx) }\end{array}$ & E-UTRAN & $P_{c, R S}^{T}$ \\
\hline $\begin{array}{l}\text { Received Interference Power } \\
\text { (RIP) }\end{array}$ & E-UTRAN & $\bar{I}_{c}^{T}$ \\
\hline
\end{tabular}
LTE measurements, and includes the notation followed in this paper for a tagged user $u$, cell $c$, and measurement interval $T$.

Table I: Link quality measurements for the LTE system [15]

Note that the $\bar{I}_{c}^{T}$ measurement in Table I corresponds to the linear average of the RIP measurements performed within the tagged cell's operating bandwidth, i.e., the utilized Resource Blocks [15]. To the remainder of this paper, we focus on the HO decision phase, which is performed in the serving LTE cells. The network discovery procedure is outside the scope of this paper, and it is assumed that for all UEs connected to it, each serving LTE cell has a consistent candidate cell set, and link quality measurements describing its status.

\section{B. Strongest cell handover decision policy}

In the context of LTE, the strongest cell HO decision policy results in a $\mathrm{HO}$ execution whenever the RSRP of an accessible cell exceeds over the RSRP of the serving cell plus a policy-defined HHM, for a policy-defined time period namely the Time To Trigger (TTT) [13]. The HHM is utilized to mitigate frequency-related propagation divergences, and the ping-pong effect. Based on our system model, the strongest cell HO policy for the LTE system is described as follows:

$$
\begin{gathered}
\arg \max _{\mathrm{c} \in \boldsymbol{L}_{\boldsymbol{u}}} R S R P_{c \rightarrow u,(d B)}^{T T T}:=\left\{c \mid R S R P_{c \rightarrow u,(d B)}^{T T T}>\right. \\
\left.R S R P_{s \rightarrow u,(d B)}^{T T T}+H H M_{c,(d B)}\right\}
\end{gathered}
$$

where $H H M_{c,(d B)}$ corresponds to the HHM for cell c $\in \boldsymbol{L}_{\boldsymbol{u}}$, and the notation $X_{(d B)}$ to the value of $X$ in decibels (dB). Taking into account the definition of the RSRP in [15], it follows that:

$$
\operatorname{RSRP}_{c \rightarrow u}^{T}=P_{c, R S}^{T} \cdot \bar{h}_{c \rightarrow u}^{T}(5)
$$

Substituting Eq. (5) to Eq. (4), it follows that the strongest cell policy facilitates mobility towards cells with higher RS power transmissions or improved channel gain. As a result, even though the strongest cell policy may improve the channel gain for the tagged LTE user (Eq. 5), it does not necessarily improves the SINR performance (Eq. 1,2), given that neither the RF interference, nor the actual RS power transmissions of the target cells, are taken into account. The same implies for the UE power transmissions, which are not necessarily being reduced (Eq. 3), having a negative impact on both the UE power consumption and the RF interference network-wide. 


\section{THE PROPOSED HANDOVER DECISION POLICY}

The proposed HO decision policy, referred to as UE Transmit Power Reduction (UTPR) policy in the following, consists of handing over to the cell with the minimum required UE transmit power, while maintaining the mean SINR target. The following analysis is pursued to derive the HHM required for minimizing the UE power transmissions, based on the available set of standard LTE measurements in Table I. It is assumed that user $u$ receives service from cell $s$, which has consistent LTE measurements describing the status of every candidate cell c $\in \boldsymbol{L}_{\boldsymbol{u}}$ for user $u$, for the time interval $T=T T T$.

Using (5) under the assumption of a symmetric channel gain, the following estimation can be made:

$$
\bar{h}_{u \rightarrow c}^{T} \cong \bar{h}_{c \rightarrow u}^{T}=\frac{R S R P_{c \rightarrow u}^{T}}{P_{c, R S}^{T}}(6)
$$

By the RIP measurement definition in [15], it follows that:

$$
\bar{I}_{c}^{T}=\left(\sum_{c^{\prime} \in C_{n}-\{c\}} \bar{P}_{c^{\prime}}^{T} \cdot \bar{h}_{c^{\prime} \rightarrow c}^{T}+\sum_{u^{\prime} \in U_{n}} \bar{P}_{u^{\prime}}^{T} \cdot \bar{h}_{u^{\prime} \rightarrow c}^{T}+\left(\bar{\sigma}_{c}^{T}\right)^{2}\right)(7)
$$

Using Eq. (3), (6), and (7), it can be shown that the UE power transmission on the serving cell $s$ is given by (8).

$$
\bar{P}_{u}^{T} \triangleq \bar{P}_{u \rightarrow s}^{T}=\frac{\bar{\gamma}_{\text {target }}^{u} \cdot P_{S, R S}^{T} \cdot \bar{I}_{S}^{T}}{\operatorname{RSRP}_{S}^{T} \rightarrow u}(8)
$$

Following a similar approach, the UE transmit power on the candidate cell $\mathrm{c}$ can be estimated as follows:

$$
\bar{P}_{u \rightarrow c}^{T}=\frac{\bar{\gamma}_{\text {target }}^{u} \cdot P_{c, R S}^{T} \cdot\left(\bar{I}_{c}^{T}-\bar{P}_{u}^{T} \cdot \bar{h}_{u \rightarrow c}^{T}\right)}{\operatorname{RSRP}_{c \rightarrow u}^{T}} \text { (9) }
$$

where the term $\bar{P}_{u}^{T} \cdot \bar{h}_{u \rightarrow c}^{T}$ is introduced to include the positive impact of handing over to cell $c \in \boldsymbol{L}_{\boldsymbol{u}}$, if cells $c$ and $s$ operate in the same LTE band (if not, it is omitted), i.e, if $c, s \in \boldsymbol{C}_{\boldsymbol{n}}$. Accordingly, handing over to the candidate cell $\mathrm{c}$, is expected to result in reduced UE transmit power compared to the one used in the current serving cell $s$, if the following are in effect:

$$
\begin{gathered}
\bar{P}_{u \rightarrow s}^{T}>\bar{P}_{u \rightarrow c}^{T} \Rightarrow(10) \\
\frac{\bar{\gamma}_{\text {target }}^{u} \cdot P_{S, R S}^{T} \cdot \bar{I}_{s}^{T}}{R S R P_{s \rightarrow u}^{T}}>\frac{\bar{\gamma}_{\text {target }}^{u} \cdot P_{c, R S}^{T} \cdot\left(\bar{I}_{c}^{T}-\bar{P}_{u}^{T} \cdot \bar{h}_{u \rightarrow c}^{T}\right)}{R S R P_{c \rightarrow u}^{T}} \Rightarrow \\
R S R P_{c \rightarrow u}^{T}>R S R P_{S \rightarrow u}^{T} \cdot \frac{P_{c, R S}^{T} \cdot\left(\bar{I}_{c}^{T}-\bar{P}_{u}^{T} \cdot \bar{h}_{u \rightarrow c}^{T}\right)}{P_{s, R S}^{T} \cdot \bar{I}_{S}^{T}}
\end{gathered}
$$

where Eq. (11) is derived by using Eq. (8), and (9), and Eq. (12) by rearranging (11). Note that the parameter $\bar{P}_{u}^{T}$ is given by Eq. (8). By taking the respective parameter values in $\mathrm{dB}$, Eq. (12) can be rearranged as follows:

$$
R S R P_{c \rightarrow u,(d B)}^{T T T}>R S R P_{s \rightarrow u,(d B)}^{T T T}+H H M_{c,(d B)}^{U T P R}
$$

where the parameter $H H M_{c,(d B)}^{U T P R}$ is given by (14).

$$
H H M_{c,(d B)}^{U T P R}=\left\{\begin{array}{cc}
10 \log \frac{P_{c, R S}^{T T T} \cdot\left(\bar{I}_{c}^{T T T}-\bar{P}_{u}^{T T T} \cdot \bar{h}_{u \rightarrow c}^{T T T}\right)}{P_{S, R S}^{T T T} \cdot \bar{I}_{S}^{T T T}} & c, s \in C_{\boldsymbol{n}} \\
10 \log \frac{P_{c, R S}^{T T T} \cdot \bar{I}_{c}^{T T T}}{P_{S, R S}^{T T T} \cdot \bar{I}_{S}^{T T T}} & \text { otherwise }
\end{array}\right.
$$

It can be seen that Eq. (13) can be utilized as a HO decision criterion for minimizing the UE power transmissions in the two-tier LTE network. To achieve this, Eq. (14) can be incorporated in the standard LTE HO procedure, as an adaptive HHM. Given that a HHM for mitigating the side-effects of user mobility is still required, the $H H M_{c,(d B)}^{U T P R}$ parameter should be incorporated as an additional HHM in the strongest cell HO decision policy. Taking this into account, the proposed UTPR HO decision policy can be described as follows:

$$
\begin{aligned}
& \arg \max _{\mathbf{c} \in \boldsymbol{L}_{\boldsymbol{u}}} R S R P_{c \rightarrow u,(d B)}^{T T T}:=\left\{c \mid R S R P_{c \rightarrow u,(d B)}^{T T T}>\right. \\
& \left.R S R P_{s \rightarrow u,(d B)}^{T T T}+H H M_{c,(d B)}+H H M_{c,(d B)}^{U T P R}\right\}
\end{aligned}
$$

Summarizing, the proposed UTPR policy is based on standard LTE measurements, while it is employed by introducing an adaptive HHM to the standard LTE HO procedure. The employment of the UTPR policy does not require any enhancements for the LTE UEs. An enhanced network signaling procedure is necessitated, however, to convey the E-UTRAN measurements amongst the cells. This signaling procedure can be based on directly exchanging the required measurement information through the standard X2 interface [2]. Alternatively, a Core Network (CN) entity can be deployed for gathering, maintaining, and disseminating the required E-UTRAN measurements on demand. This $\mathrm{CN}$ entity can also control the E-UTRAN measurement signaling load, i.e., LTE measurement requests and reports, depending on the current CN load, the LTE cells' status, and other networkrelated parameters. The details of the required network signaling procedure are omitted, and left as future work.

\section{NUMERICAL RESULTS}

This section includes selected numerical results to evaluate the performance of the proposed HO decision policy in the two-tier LTE network. The simulation scenario is based on the evaluation methodology described in [16], while the proposed UTPR policy is compared against the strongest cell $\mathrm{HO}$ decision policy, referred to as SC policy in the following.

A conventional hexagonal LTE network is considered, including a main LTE cluster composed of 7 LTE cells, where each LTE cell consists of 3 hexagonal sectors. The wraparound technique is used to extend the LTE network, by copying the main LTE cluster symmetrically on each of the 6 sides. A set of blocks of apartments, referred to as femtoblocks, are uniformly dropped within the main LTE cluster according to the parameter $d_{F B}$, which indicates the femtoblock deployment density within the main LTE cluster, i.e., the percentage of the main LTE cluster area covered with femtoblocks. Each femtoblock is modeled according to the dual stripe model for dense urban environments in [16]. According to it, each femtoblock consists of two stripes of apartments separated by a $10 \mathrm{~m}$ wide street, while each stripe has two rows of $A=5$ apartments of size $10 \times 10 \mathrm{~m}$. For a tagged femtoblock, femtocells are deployed with a femtocell deployment ratio parameter $r_{f c}$, which indicates the percentage of apartments with a femtocell [16]. Each femtocell initially serves one associated user, while in general, it can serve up to 4 users. Femtocells and femtocell users are uniformly dropped inside the apartments. Each LTE user is member of up to one 
CSG, where the CSG ID per user and femtocell is uniformly picked from the set $\{1,2,3\}$. Each LTE sector initially serves ten macrocell users, which are uniformly distributed within it. The user mobility model consists of two parameters: the user speed $v_{t}$ and the user direction $\varphi_{t}[8]$, which are updated by:

$v_{t}=N\left(\bar{v}, s_{u}\right)$ and $\varphi_{t}=N\left(\varphi_{t-1}, 2 \pi-\varphi_{t-1} \tan \left(\frac{\sqrt{v_{t}}}{2}\right) \Delta t\right)$

where $\bar{v}$ indicates the mean user speed, $s_{u}$ the speed standard deviation, $\Delta t$ the time between two updates of the model, and $N(a, b)$ the Gaussian distribution [8]. Unless differently stated, it is assumed that $\bar{v}=3 \mathrm{~km} / \mathrm{h}$ and $s_{u}=1 \mathrm{~km} / \mathrm{h}$.

The macrocell stations operate in a LTE band centered at 2 $\mathrm{GHz}$, utilizing a $5 \mathrm{MHz}$ bandwidth. The macrocell inter-site distance is set to $500 \mathrm{~m}$. The operating band for each femtocell is uniformly picked from a band set including the macrocell operating band and the two adjacent frequency bands of $5 \mathrm{MHz}$ bandwidth. The minimum required SINR per UE is set to $\bar{\gamma}_{t}^{u}=3 \mathrm{~dB}$, while the communications are carried out in full buffer [16]. The shadowing standard deviation for the macrocell and femtocell stations are 8 and $4 \mathrm{~dB}$, respectively, and the noise figures are set to 5 and $8 \mathrm{~dB}$ in that order. The macrocell downlink RS power transmissions are normally distributed with a mean of $23 \mathrm{dBm}$ and standard deviation $3 \mathrm{~dB}$, while the femtocell downlink RS power transmissions are uniformly distributed within the $[0,10] \mathrm{dBm}$ interval. The UE power class is set to $23 \mathrm{dBm}$, and the maximum transmit power for the macrocell and femtocell stations are set to 43 and 10 $\mathrm{dBm}$, respectively. The path loss is described by the models for the dual stripe layout [16], while the frequency-selective fading is Rayleigh distributed [8]. Finally, the overall simulation time is set to $1000 \mathrm{sec}$ with a simulation unit of $\Delta t=1 \mathrm{sec}$.

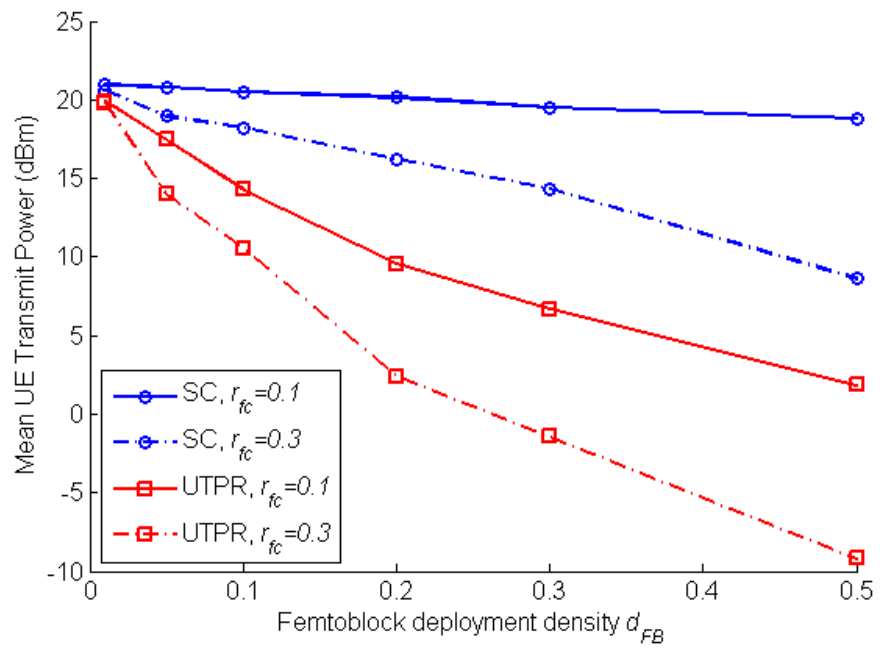

Figure 1: Mean UE transmit power versus the femtoblock deployment density

Fig. 1 depicts the performance of the SC and UTPR policies in terms of mean UE transmit power. As expected, an increased femtoblock deployment density $d_{F B}$ results in lower UE transmit power for both policies. However, a higher femtocell deployment density $r_{f c}$ is required for the SC policy to lower the UE transmit power. On the contrary, the UTPR policy results in comparably lower UE transmit power, even for low $r_{f c}$, especially when a higher femtoblock deployment density characterizes the network layout. This improvement originates from the incorporation of the actual downlink RS and received interference power at the cell sites, while it varies from 1 to $20 \mathrm{~dB}$, depending on the femtoblock and the femtocell deployment density. Given that the transmit power is the main UE power consumption contributor, this improvement corresponds to an equally reduced UE power consumption, too.

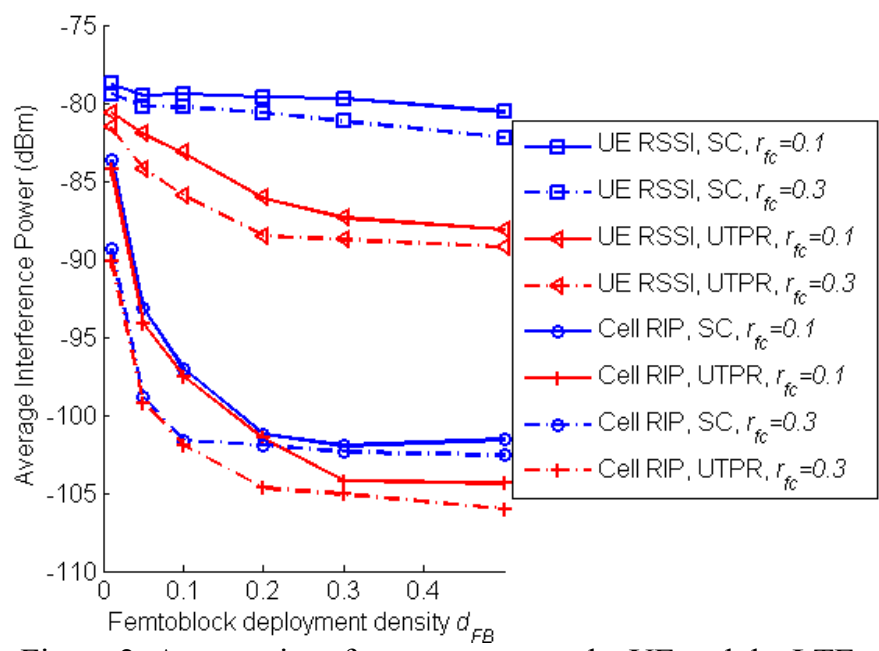

Figure 2: Average interference power at the UE and the LTE cell sites, versus the femtoblock deployment density

Fig. 2 illustrates that the proposed UTPR policy greatly lowers the Received Interference Power as well, both in terms of RSSI at the UEs (up to $8 \mathrm{~dB}$ ) and RIP at the LTE cell sites (up to $3 \mathrm{~dB}$ ). This positive impact originates from the proposed policy's tendency in handing over to cells with lower RIP, leading to reduced number of UE interferers in congested bands and lower UE transmit power per band network-wide.

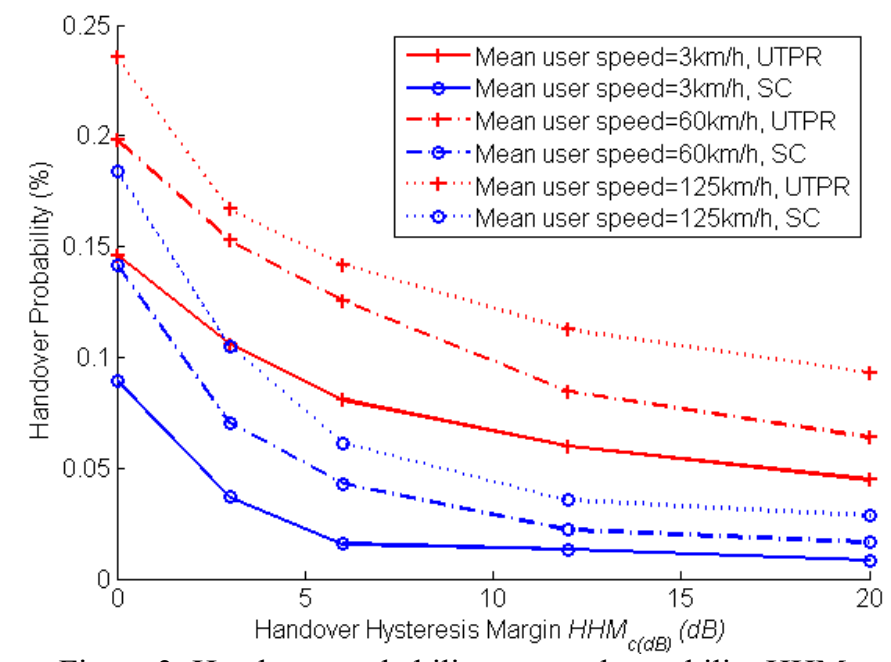

Figure 3: Handover probability versus the mobility HHM

Fig. 3 plots the HO probability for varying mobility HHM, i.e, $H H M_{c,(d B)}$ parameter in Eq. (4), and (13). The results are derived for $d_{F B}=0.05, r_{f c}=0.2$, and three different mean user speeds, i.e., 3, 60 and $125 \mathrm{~km} / \mathrm{h}$. It can be seen that although the employment of the proposed UTPR policy greatly lowers the UE power consumption owing to transmit power (Fig. 1), and the network-wide RF interference (Fig. 2), an increased $\mathrm{HO}$ probability is observed compared to the SC 
policy (Fig. 3). This negative impact originates from a) the proposed policy's tendency to extend the femtocell utilization time, which results in increased sensitiveness on user mobility, and $b$ ) the fact that the proposed policy takes into account the $\mathrm{RF}$ interference at the cell sites, which is in general object to more variations compared to the RS power transmissions and the channel gain (SC policy). Fig. 3 also shows that an increased $H H M_{c,(d B)}$ value can lower the $\mathrm{HO}$ probability for the UTPR policy, at comparable levels with the SC policy. An increased $H H M_{c,(d B)}$ value, however, also comes with reduced potential to lower the UE power transmissions for the proposed HO policy (Fig. 4). Nevertheless, the UTPR policy still results in significantly lower UE transmit power, varying from 3 to 8 $\mathrm{dB}$ for the scenario under consideration, depending on the mean user speed and the adopted $H H M_{c,(d B)}$ value. Note that the proposed policy lowers the UE transmit power even in high user speeds, where the femtocell tier is not utilized, owing to the proposed policy's capability to take into account the RF interference level at the macrocell sites. This positive impact follows from the random femtocell deployment pattern, which results in divergent RF interference to the LTE macrocells.

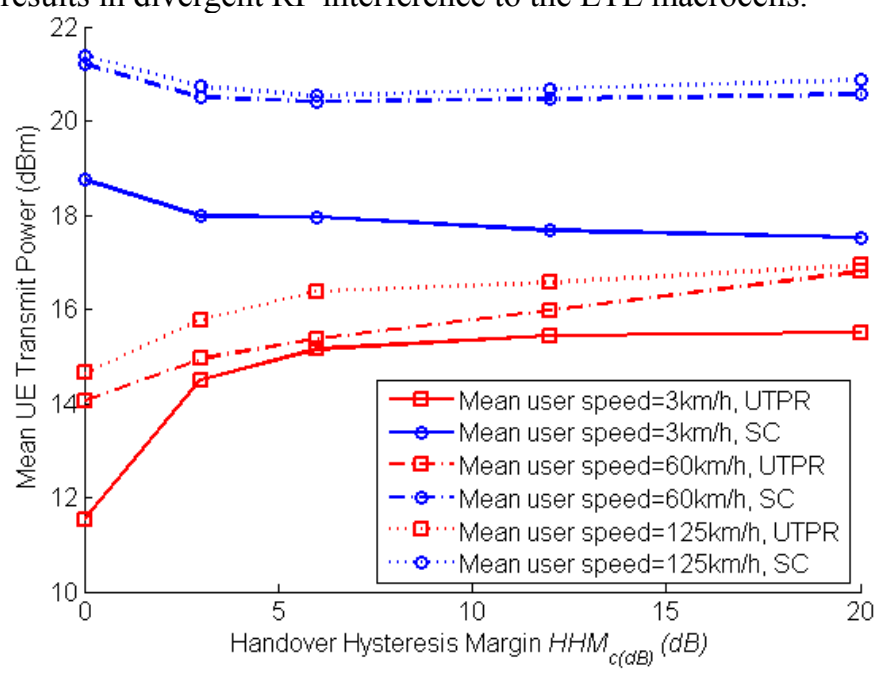

Figure 4: Mean UE transmit power versus the HHM

\section{CONCLUSION}

The random femtocell deployment may result in degraded SINR performance, increased outage probability, and enlarged network signaling, if the interference-agnostic strongest cell policy is employed during the $\mathrm{HO}$ decision phase. This paper introduced a novel HO decision policy for reducing the UE power transmissions in the two-tier LTE network, while attaining a prescribed SINR target for the LTE users. This policy is fundamentally different from the strongest cell $\mathrm{HO}$ policy, as it takes into account the RS power transmissions and the RF interference at the LTE cell sites. The proposed policy is LTE backwards compatible, as it is employed by adapting the HHM with respect to the user's mean SINR target and standard link quality measurements describing the status of the candidate LTE cells. Even though employing the proposed policy necessitates an enhanced network signaling between cells, numerical results demonstrate greatly lower networkwide RF interference, and reduced UE power consumption owing to transmit power, compared to the strongest cell $\mathrm{HO}$ policy. The impact of using an increased HHM for mobility mitigation has also been investigated, both in terms of $\mathrm{HO}$ probability, and power saving potential. Future work includes a detailed signaling procedure for employing the UTPR policy, and a UTPR-based HO decision algorithm, to further enhance the effectiveness of the HO decision phase in LTE-Advanced.

\section{ACKNOWLEDGMENT}

This paper has been partially funded by the C2POWER (FP7-ICT- 248577), GREEN-T (CP8-6), GREENET (FP7PEOPLE 264759), CO2GREEN (TEC2010-20823) projects, and co-financed by the EU (European Social Fund - ESF) and Greek national funds through the Operational Program "Education and Lifelong Learning" of the National Strategic Reference Framework (NSRF) - Research Funding Program: Heracleitus II. Investing in knowledge society through the European Social Fund.

\section{REFERENCES}

[1] V. Chandrasekhar, J. Andrews, A. Gatherer, "Femtocell networks: a survey”, IEEE Commun. Magazine, vol.46, no.9, pp.59-67, Sept. 2008.

[2] 3GPP, "E-UTRA and E-UTRAN Overall Description.", TS 36.300 V10.4.0, June 2011.

[3] H. Leem, S. Y. Baek, D. K. Sung, "The Effects of Cell Size on Energy Saving, System Capacity, and Per-Energy Capacity," IEEE Wireless Communications and Networking Conference, pp.1-6, Apr. 2010.

[4] F. Cao, Z. Fan, "The tradeoff between energy efficiency and system performance of femtocell deployment," The 7th Internat. Symposium on Wirel. Commun. Syst. (ISWCS), pp.315-319, Sept. 2010.

[5] M. Yavuz, F. Meshkati, S. Nanda, A. Pokhariyal, N. Johnson, B. Raghothaman, A. Richardson, "Interference management and performance analysis of UMTS/HSPA+ femtocells," IEEE Communications Magazine, vol.47, no.9, pp.102-109, Sept. 2009.

[6] V. Chandrasekhar, J.G. Andrews, T.Muharemovic, Z. Shen, A. Gatherer, "Power control in two-tier femtocell networks," IEEE Transactions on Wireless Communications, vol.8, no.8, pp.4316-4328, Aug. 2009.

[7] G. Boudreau, J. Panicker, G. Ning, R. Chang, N. Wang, S. Vrzic, "Interference coordination and cancellation for 4G networks," IEEE Communications Magazine, vol.47, no.4, pp.74-81, Apr. 2009.

[8] J. Zhang, G. de la Roche, "Femtocells : technologies and deployment", John Wiley \& Sons Ltd, ISBN 978-0-470-74298-3, 2010.

[9] W. Shaohong, Z. Xin, Z. Ruiming, Y. Zhiwei, F. Yinglong, Y. Dacheng, "Handover Study Concerning Mobility in the Two-Hierarchy Network," IEEE 69th Vehicular Technology Conference, pp.1-5, Apr. 2009.

[10] A. Ulvan, R. Bestak, M. Ulvan, "Handover Scenario and Procedure in LTE-based Femtocell Networks", The 4th International Conference on Mobile Ubiquitous Comput., Syst., Serv. and Technolog., Oct. 2010.

[11] S. Sesia, I. Toufik, M. Baker, "LTE - The UMTS Long Term Evolution: From Theory to Practice", John Wiley \& Sons, ISBN: 978-0-470-69716$0,2009$.

[12] Z. Becvar, P.Mach, "Adaptive Hysteresis Margin for Handover in Femtocell Networks", 6th International Conference on Wireless and Mobile Communications, pp.256-261, Sept. 2010.

[13] K. Dimou, M. Wang, Y. Yang, M. Kazmi, A. Larmo, J. Pettersson, W. Muller, Y. Timner, "Handover within 3GPP LTE: Design Principles and Performance," IEEE 70th Vehicular Technology Conference Fall, pp.15, Sept. 2009.

[14] I. Ashraf, L.T.W. Ho, H. Claussen, "Improving Energy Efficiency of Femtocell Base Stations Via User Activity Detection," IEEE Wireless Communications and Networking Conference 2010, pp.1-5, Apr. 2010.

[15] 3GPP, "Physical layer; Measurements", TS 36.214 V10.1.0, Mar. 2011.

[16] Femto Forum, "Interference Management in OFDMA Femtocells", Femto Forum, Mar. 2010.

[17] 3GPP, "LTE physical layer; General description", TS 36.201 V10.1.0 (2010-12). 